\title{
Relative contribution of Prevotella intermedia and Pseudomonas aeruginosa to lung pathology in airways of patients with cystic fibrosis
}

\author{
Martina Ulrich, ${ }^{1}$ Isabelle Beer, ${ }^{1}$ Peter Braitmaier, ${ }^{1}$ Michaela Dierkes, ${ }^{1}$ \\ Florian Kummer, ${ }^{1}$ Bernhard Krismer, ${ }^{1}$ Ulrike Schumacher, ${ }^{1}$ Ute Gräpler-Mainka, ${ }^{2}$ \\ Joachim Riethmüller, ${ }^{2}$ Peter $\emptyset$ Jensen, ${ }^{3}$ Thomas Bjarnsholt, ${ }^{3}$ Niels Høiby, ${ }^{3}$ \\ Gabriel Bellon, ${ }^{4}$ Gerd Döring ${ }^{1}$
}

\begin{abstract}
- Additional materials are published online only. To view these files, please visit the journal online (http://thorax.bmj. com)

${ }^{1}$ Institute of Medical Microbiology and Hygiene, Universitätsklinikum Tübingen, Tübingen, Germany

${ }^{2}$ Kinderklinik,

Universitätsklinikum Tübingen, Tübingen, Germany

${ }^{3}$ Department of Clinical Microbiology, Rigshospitalet, Copenhagen, Denmark ${ }^{4}$ Groupement Hospitalier Est Hôpital Femme Mère Enfant, Bron, France
\end{abstract}

\section{Correspondence to}

Gerd Döring, Institute of Medical Microbiology and Hygiene, Universitätsklinikum Tübingen, Wilhelmstrasse 31, D-72074 Tübingen, Germany; gerd. doering@med.uni-tuebingen.de

Received 25 February 2010 Accepted 23 July 2010

Published Online First 29 September 2010

\begin{abstract}
Background Patients with cystic fibrosis (CF) with Pseudomonas aeruginosa lung infections produce endobronchial mucus plugs allowing growth of obligate anaerobes including Prevotella spp. Whether obligate anaerobes contribute to the pathophysiology of CF lung disease is unknown.
\end{abstract}

Methods The virulence of Prevotella intermedia and $P S$ aeruginosa was investigated in vitro and in mice, antibodies against $P$ intermedia in CF sera were assessed and a culture-independent detection method for $P$ intermedia/P nigrescens in CF sputum was tested. Results $P$ intermedia reached cell numbers of $>10^{5}->10^{7}$ colony-forming units (CFU)/ml sputum. The majority of patients with CF (16/17; 94.1\%) produced antibodies against two immunoreactive antigens of $P$ intermedia. Culture supernatant fluids, collected from $10^{9}$ $P$ intermedia cells, were more cytotoxic to respiratory epithelial cells in vitro and inflammatory in mouse lungs than respective fluids from anaerobically grown $P S$ aeruginosa, while fluids from aerobically grown $P_{S}$ aeruginosa had the highest cytotoxicity and inflammation. Both pathological effects were largely reduced when culture supernatant fluids from $10^{7}$ cells of either species were used. $P$ intermedia cells ( $\sim 10^{6} \mathrm{CFU} /$ lung) did not induce mortality in the agar beads lung infection mouse model, while $P$ s aeruginosa cells caused death in $30 \%$ of mice due to rapid multiplication. A P intermedia/P nigrescens-specific PNA probe was significantly more sensitive than culturedependent diagnostic assays to detect these strict anaerobes.

Conclusion $P$ s aeruginosa and $P$ intermedia become significantly virulent in vitro and in vivo when cell numbers exceed $10^{8} \mathrm{CFU} /$ lung.

\section{INTRODUCTION}

In patients with cystic fibrosis (CF), chloride retention as a consequence of defective CF transmembrane conductance regulator and increased sodium and water absorption causes an abnormally high viscosity of the mucus layer on the respiratory epithelium. $^{12}$ The resulting impaired mucociliary clearance initiates a vicious circle of chronic infection and inflammation in the airways of patients with $\mathrm{CF}^{3}$ Epidemiological data from a large patient registry have shown that the facultative anaerobic bacteria Staphylococcus aureus and Pseudomonas aeruginosa are the dominating pathogens in $\mathrm{CF}$ lung disease. ${ }^{4}$ Both pathogens form biofilms under hypoxic conditions, ${ }^{5-8}$ thereby resisting host defence and antibiotic therapy. ${ }^{9} 10$ The persistence of facultative anaerobic bacteria results in a continuous influx of neutrophils into the airway lumen ${ }^{3}$ and, particularly in the absence of effective antibiotic therapy, large hypoxic mucus plugs are formed which lead to considerable airway obstruction

Rapid consumption of oxygen by facultative anaerobic pathogens ${ }^{8}$ and by the large number of luminal neutrophils ${ }^{11}$ may favour substantial growth of obligate anaerobes. With the exception of two early reports, ${ }^{12}{ }^{13}$ obligate anaerobes in CF have only recently gained increased interest. Many obligate anaerobe species have been detected in CF sputum samples. ${ }^{14-20}$ High cell numbers of these anaerobes have ruled out the notion that the presence of these microorganisms in sputum is derived from upper airway contamination. ${ }^{16} 17$ The oral cavity has been regarded as a 'stepping stone' for lung colonisation and infection for certain bacterial anaerobic species. ${ }^{16}$

However, the important question-whether obligate anaerobes contribute to the pathophysiology of lung disease in CF-is still unresolved. In addition, it remains to be investigated whether widely used culture-dependent identification methods for obligate anaerobes correctly reflect the presence of obligate anaerobes in CF sputum.

We have addressed these issues by focusing on Prevotela intermedia. This Gram-negative blackpigmented anaerobic rod has been repeatedly detected in CF airway specimens. ${ }^{17-20}$ We comparatively investigated the virulence of $P$ intermedia and $P_{S}$ aeruginosa in vitro and in mice, assessed antibodies against $P$ intermedia in CF serum and tested a culture-independent detection method for $P$. intermedia, $P$. nigrescens and P. melaninogenica in CF sputum.

\section{METHODS \\ Patients}

Serum and sputum samples and lung function data were collected from 17 patients with CF attending the CF centre of the University Children's Clinic, Universitätsklinikum Tübingen, Tübingen, Germany. Patients were eligible if they were infected with $P_{S}$ aeruginosa and were sputum producers. To obtain a representative sputum 
sample, at least $2 \mathrm{ml}$ of purulent sputum had to be produced by the patients during routine visits to the CF centre in Tübingen. Serum samples from 30 healthy individuals were also collected. Healthy individuals were recruited from members of staff at the University Hospital of Tübingen. In addition, four patients with CF who attended the CF centre of the Service de Pediatrie, Centre Hospitalier Lyon-Sud, Pierre-Benite, France were studied. ${ }^{21}$

\section{Identification of Prevotella species}

Bronchopulmonary samples for bacteriological investigations including sputum, bronchial aspiration, protected catheter brushing, mucosal biopsies and bronchial lavages were processed by routine methods as described in the online supplement. Prevotella species were identified using API 32A, a fluoresceinlabelled 15-mer PNA probe by in situ hybridisation, MALDITOF-MS and sequencing (for details see online supplement). ${ }^{22} 23$

\section{Antibody response to $\boldsymbol{P}$ intermedia antigens in CF serum}

Serum samples from patients with CF were subjected to western blotting and ELISA using isolated membrane proteins of the $P$ intermedia strain ATCC 25611 (for details see online supplement). ${ }^{24}$

\section{Cytotoxicity assays}

Culture supernatant fluids from $P$ s aeruginosa strain PAO1 grown under aerobic and anaerobic conditions and $P$ intermedia strain ATCC 25611 grown under anaerobic conditions were incubated with the human alveolar type II cell line A549 for $1 \mathrm{~h}$ at $37^{\circ} \mathrm{C}$. Cytotoxicity was assessed using the Syto13/propidium iodide viability test. ${ }^{25}$ Additionally, the culture supernatant fluids were incubated for various time periods with purified human polymorphonuclear leucocytes. ${ }^{26}$ Finally, the ability of the P intermedia strain ATCC 25611 to degrade casein, elastin or gelatin was assessed on agar plates with the respective substrates (for details see online supplement).

\section{Animal studies}

For lung infection of $\mathrm{C} 57 \mathrm{Bl} / 6$ mice with $P$ s aeruginosa or $P$ intermedia the agar bead model was used. ${ }^{27}$ Twenty-four hours after challenge, lungs were excised and bacterial colonyforming units (CFUs) were determined using routine methods. Mice were also challenged with sterile culture supernatant fluids from the microorganisms. In both experiments, neutrophils and macrophages were quantified in lung tissue sections. All animal experiments were approved in advance by the Regierungspräsidium Tübingen, Germany (for details see online supplement).

\section{Statistical analysis}

For statistical evaluations the Spearman correlation coefficient $\rho$, Wilcoxon/Kruskal-Wallis test, $\chi^{2}$ test and Fisher exact test were used (for details see online supplement).

\section{RESULTS}

Prevotella intermedia was selected as the prototype organism for these investigations because it has been identified as one of the most frequent obligate anaerobe in CF sputum cultures by us and others. In our study, $38 \%$ of the patients harboured Pintermedia.

\section{Culture supernatant fluids of $\boldsymbol{P}$ intermedia are cytotoxic to airway epithelial cells and neutrophils}

Microscopic evidence showed that undiluted culture supernatant fluids of $P$ intermedia corresponding to $7.8 \times 10^{8} \mathrm{CFU}$ caused cell damage in the A549 monolayer including cell rounding after $1 \mathrm{~h}$ of incubation followed by complete cell detachment (figure 1A, B). Cell death was visible in $47.5 \%$ of A459 cells after incubation for $1 \mathrm{~h}$ at $37^{\circ} \mathrm{C}$ when examined using a live/dead fluorescent staining method. When the culture supernatant fluids of $P$ intermedia had been diluted 1:10 and 1:100, the cell death rate decreased to $34.8 \%$ and $10.3 \%$ (figure $1 \mathrm{~A}, \mathrm{~B}$ ). Because $P$ intermedia strains produce various proteases, ${ }^{28}{ }^{29}$ we tested the culture supernatant fluid of the $P$ intermedia stain ATCC 25611 for degradation of gelatin, elastin and casein. In contrast to $P$ s aeruginosa strain PAO1 grown anaerobically or aerobically, undiluted culture supernatant fluids of $P$ intermedia at comparable cell numbers did not have activity against these substrates (data not shown).

Culture supernatant fluids from $P$ s aeruginosa grown anaerobically corresponding to $7.8 \times 10^{8} \mathrm{CFU}$ had a low cytotoxic effect (figure 1A,B). Culture supernatant fluids from aerobically grown $P$ s aeruginosa were significantly more cytotoxic $\left(p=1.53 \times 10^{-9}-2.21 \times 10^{-12}, \chi^{2}\right.$ test/Fisher exact test; figure $\left.1 \mathrm{~A}, \mathrm{~B}\right)$. A 1:100 dilution produced cytotoxicity of $16.3 \%$ towards A549 cells. A similar result was obtained when the culture supernatant fluids were collected when the culture was grown aerobically to a cell number of $7.2 \times 10^{8} \mathrm{CFU} / \mathrm{ml}$ (cytotoxicity $13.5 \%$ ). This cytotoxicity is significantly lower than undiluted culture supernatant fluids of $P$ intermedia $\left(p=8.08 \times 10^{-40}-1.35 \times 10^{-42}\right)$. Undiluted culture supernatant fluids from anaerobically grown Ps aeruginosa were significantly less cytotoxic than those from $P$ intermedia $\left(\mathrm{p}=9.08 \times 10^{-40}-1.44 \times 10^{-52}\right)$.

When purified human neutrophils were incubated with undiluted culture supernatant fluids from $P$ intermedia, flow cytometry showed an increasing percentage of necrotic neutrophils with time, reaching $\sim 50 \%$ dead cells after 60 min of incubation, corroborating the results obtained with A549 cells (figure 1C). Undiluted Ps aeruginosa culture supernatant fluids from strain PAO1 grown anaerobically were significantly less cytotoxic than culture supernatant fluids from $P$ intermedia $(p=0.003)$ and killed only $22 \%$ of the cells (figure 1C). In contrast, culture supernatant fluids from aerobically grown $P_{S}$ aeruginosa were highly cytotoxic and killed $99 \%$ of neutrophils within $1 \mathrm{~min}$ of incubation (figure 1C). In further experiments the inflammatory potential of culture supernatant fluids of $P$ intermedia and $P$ s aeruginosa were compared in mouse lungs (figure $1 \mathrm{D}, \mathrm{E}) . P$ intermedia and $P$ s aeruginosa caused a significantly higher influx of neutrophils $(\mathrm{p}<0.025)$ (figure 1D) and macrophages $(p<0.025)$ (figure $1 \mathrm{E})$ than anaerobically grown $P$ s aeruginosa.

Collectively, these in vitro and animal data show that the cytotoxicity of $P$ intermedia and anaerobically grown Ps aeruginosa is significantly different. They further reveal that 1:100 diluted culture supernatant fluids from both pathogens, equivalent to cell numbers of $\sim 10^{7} \mathrm{CFU}$ and irrespective of growth conditions, are markedly reduced.

\section{Virulence of $\boldsymbol{P}$ intermedia in mouse lungs is dependent on high growth rates}

When groups of mice were challenged with $3 \times 10^{6} \mathrm{CFU}$ $P$ intermedia embedded in agar beads, bacterial CFUs remained stable after $24 \mathrm{~h}$ (figure $2 \mathrm{~A}$ ). None of the mice died $48 \mathrm{~h}$ after infection. In contrast, agar bead-embedded $P S$ aeruginosa grew $1.1 \times 10^{6}-1.5 \times 10^{10} \mathrm{CFU}$ within $24 \mathrm{~h}$ after challenge (figure $2 \mathrm{~A}$ ) and killed $4 / 12(30 \%)$ of the mice after $48 \mathrm{~h}$. Similar to their differential capacity to induce mortality in mice, $P$ intermedia and $P$ s aeruginos a cells differed in their inflammatory potential in mouse lungs (figure $2 \mathrm{~B}, \mathrm{C}$ ). Washed $P$ intermedia cells caused less 

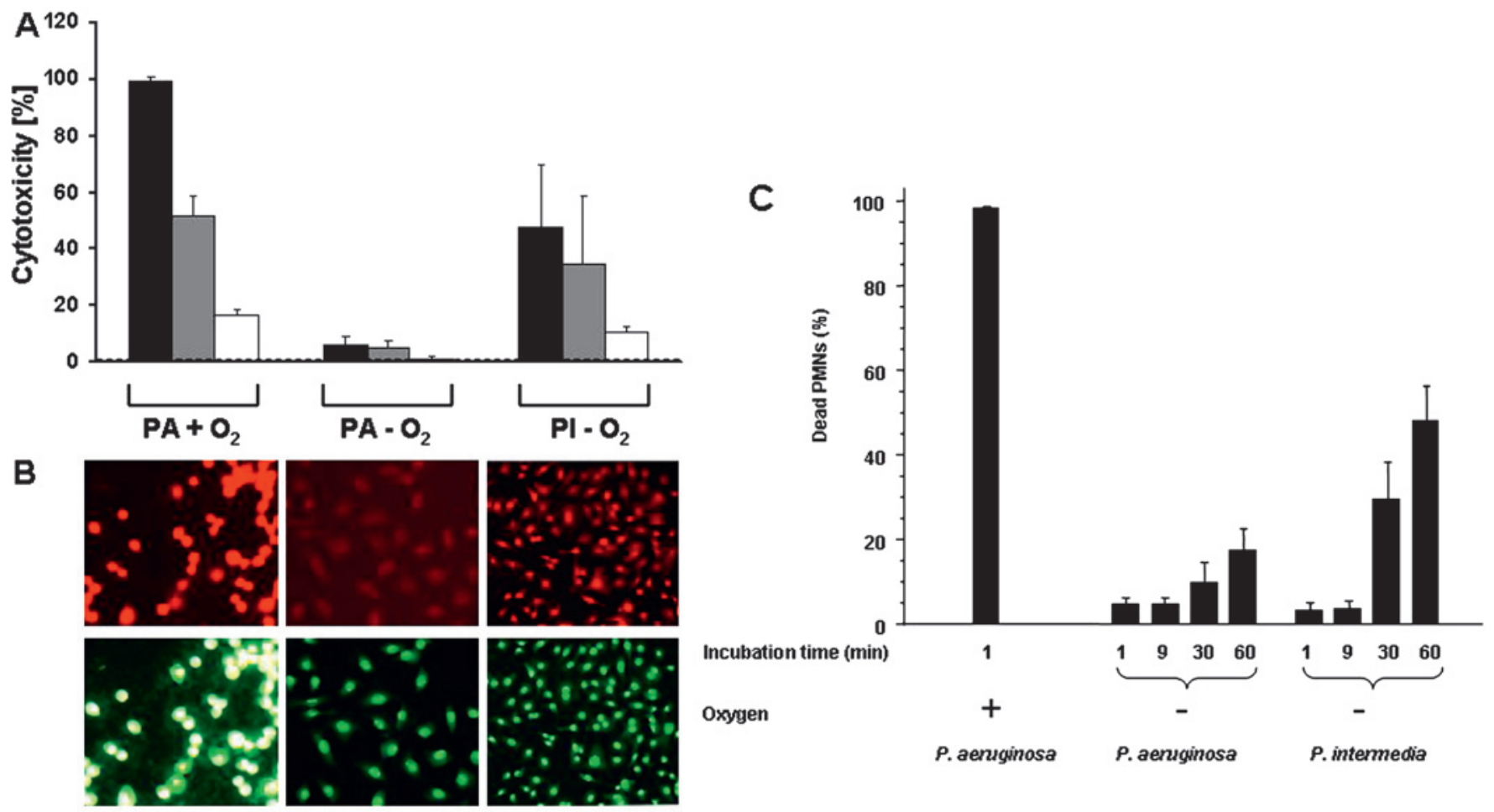

\section{Incubation time (min)}

Oxygen

$\mathrm{PI}-\mathrm{O}_{2}$
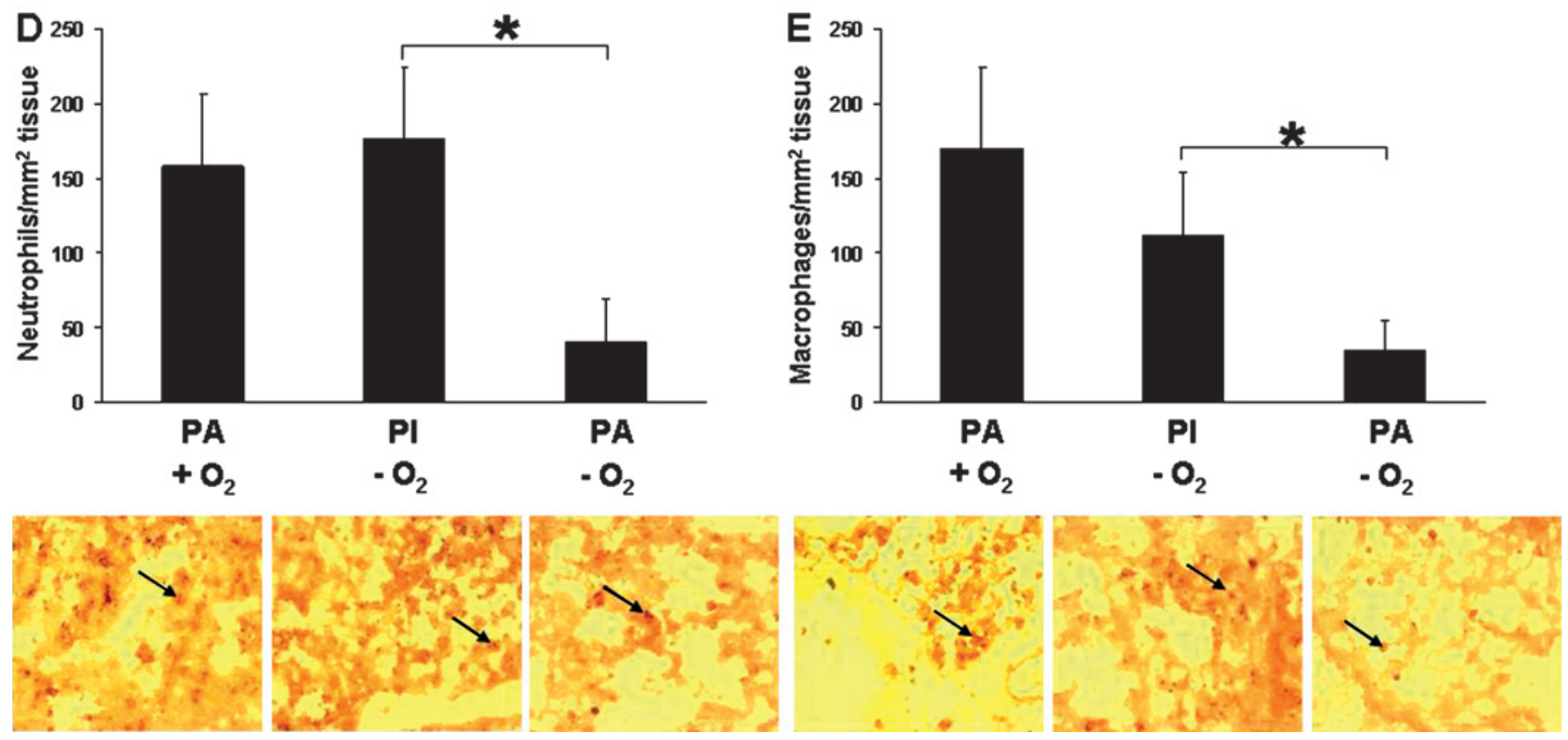

Figure 1 Prevotella intermedia produces more extracellular toxins than Pseudomonas aeruginosa under anaerobic conditions. (A, B) Cytotoxic effects of culture supernatant fluids (black bars) and diluted culture supernatant fluids (1:10, grey bars and 1:100, white bars) of $P$ intermedia (PI) grown anaerobically $\left(-0_{2}\right)$ and $P S$ aeruginosa (PA) grown anaerobically $\left(-0_{2}\right)$ or aerobically $\left(+0_{2}\right)$ on the respiratory epithelial cell line A549 determined using propidium iodide (dead cells: red) and syto13 (all cells: green). The 1:100 diluted culture supernatant fluid of Ps aeruginosa grown aerobically corresponds to a cell number of $8 \times 10^{8} \mathrm{CFU} / \mathrm{ml}$ and is comparable to the undiluted culture supernatant fluids of anaerobically grown $P$ s aeruginosa and $P$ intermedia $\left(7.8 \times 10^{8} \mathrm{CFU} / \mathrm{ml}\right.$ each). Data were obtained from 4 or 5 independent experiments which were performed in triplicate. (C) Cytotoxic effects of culture supernatant fluids of PI or PA on neutrophils (PMN) from five healthy human subjects expressed as the percentage of live neutrophils. Data were obtained from three independent experiments performed in triplicate. (D,E) Neutrophil and macrophage numbers in lung tissue of six C57BI/6 mice and the representative pictures of lung tissue sections from $\mathrm{C} 57 \mathrm{BI} / 6$ mice intratracheally challenged with culture supernatant fluids of PI or PA. Lung tissue sections were stained with specific antibodies against mouse neutrophils (D) or macrophages (E). Arrows depict positive staining. Original magnification $\times 100 .{ }^{*} \mathrm{p}<0.025$.

influx of neutrophils (figure 2B) and macrophages (figure 2C) than $P$ s aeruginosa cells within $24 \mathrm{~h}$ of the challenge. Our in vitro data showed that $P$ intermedia can grow outside agar beads when anaerobic growth conditions prevail and reach cell numbers of $2.1 \times 10^{8} \pm 9.5 \times 10^{7} \mathrm{CFU} / \mathrm{ml}$ while, under aerobic growth conditions, cell numbers remained at $3.7 \times 10^{6} \pm 4.9 \times 10^{5} \mathrm{CFU} / \mathrm{ml}$. 
A

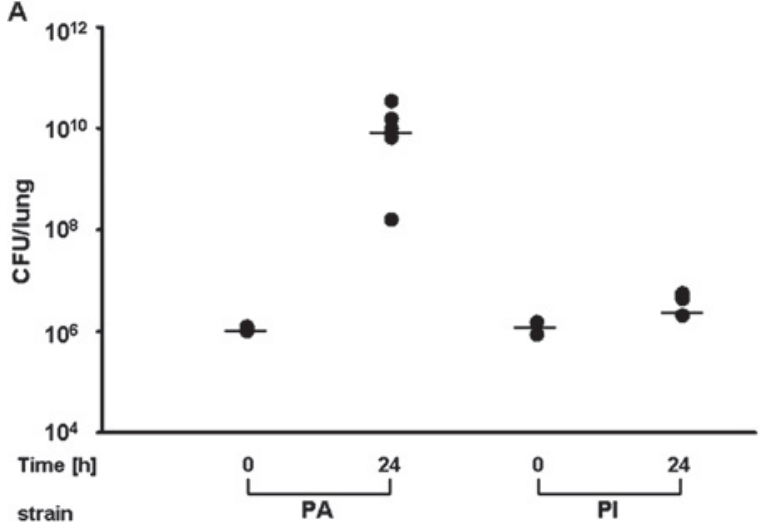

B

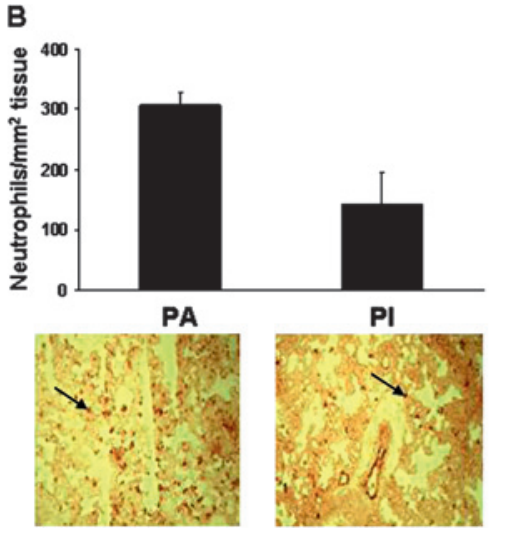

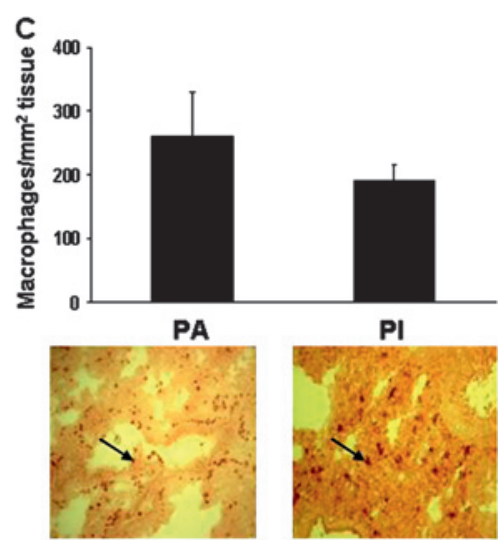

Figure 2 Prevotella intermedia cells are less virulent than Pseudomonas aeruginosa cells grown under anaerobic conditions in vivo. (A) Mice were challenged with $P$ s aeruginosa (PA) or $P$ intermedia (PI) embedded in anaerobic agar beads and bacterial cell numbers (CFU) were determined in lung tissues at time 0 and $24 \mathrm{~h}$. (B) Neutrophil and (C) macrophage numbers in lungs of mice challenged with PA or PI for $24 \mathrm{~h}$ determined with immunohistochemistry. Arrows depict positive staining. Original magnification $\times 100$.

These data suggest that $P$ intermedia contributes to lung pathology if appropriate growth conditions are present which allow multiplication to high cell numbers ( $\left.>10^{8} \mathrm{CFU} / \mathrm{lung}\right)$. This suggestion needs to be validated in further studies.

\section{$\boldsymbol{P}$ intermedia is recognised by specific serum antibodies in patients with CF}

To investigate whether the P intermedia in CF sputum samples is recognised by the adaptive immune system of patients, as has been investigated for Ps aeruginosa, ${ }^{30}{ }^{31}$ we determined antibody titres against $P$ intermedia in serum samples from patients with $\mathrm{CF}$ and healthy individuals using a newly developed ELISA (figure 3A). Cohorts comprised 17 patients with CF (median age 15 years) and 30 healthy individuals (median age 25 years). A cutoff at a reciprocal titre of 510 in the ELISA was determined as three times the unspecific binding value of a group of healthy human individuals. Patients with CF harboured positive antibody titres against $P$ intermedia antigens in $94.1 \%$ of serum samples while none of the healthy control serum samples was positive. Thus, IgG antibody titres were significantly increased in patients with CF compared with healthy individuals $(p<0.001)$.

Western blotting and MALDI-TOF showed that serum antibodies from patients with CF but not antibodies from healthy individuals bind to a $64 \mathrm{kD}$ protein (PIN A0373) with hypothetical function and to a $42 \mathrm{kD}$ immunoreactive protein (PIN A0102) (figure 3B). In order to prevent unspecific Fc-mediated binding of the antibodies to PIN A0373 and PIN A0102, we blocked the membrane with $17 \mu \mathrm{g} / \mathrm{ml}$ of the Fc fragment of human IgG. The western blot results were corroborated when crossed immunoelectrophoresis was used to determine the number of different antibodies in serum samples from our CF population. In two high-titre serum samples only two antibody arcs against single $P$ intermedia cell antigens were visible (figure $3 \mathrm{C}$ ); in contrast, $P_{S}$ aeruginosa provoked the production of up to 64 different antibodies during chronic infection in patients with CF (figure 3D). A weak non-correlation $(\rho=-0.253$, p $>0.001$ ) was obtained between antibody titres against $P$ intermedia cell proteins and lung function in patients with CF. The data suggest that $P$ intermedia is recognised by the humoral immune response in patients with CF.

\section{Numbers of strict anaerobic bacteria in CF bronchopulmonary samples}

Tunney and colleagues have determined the numbers of anaerobes in sputum specimens from patients with $\mathrm{CF}^{17}$ They reported total viable counts of Prevotella species of $>10^{4}-10^{7} \mathrm{CFU} / \mathrm{g}$
Figure 3 Immune recognition of Prevotella intermedia in patients with cystic fibrosis (CF). (A) ELISA: IgG antibody titres against $P$ intermedia cell proteins in $17 \mathrm{CF}$ and 30 control (N) serum samples. Reciprocal cut-off titre 510 ( ${ }^{*} \mathrm{p}<0.001$ ). (B) Western blot: IgG antibodies in serum from two patients with CF (CF1, CF2) but not from two healthy individuals (N1, N2) reacted with two membrane proteins of $P$ intermedia identified as PIN A0373 and PIN A0102. (C, D) Crossed immunoelectrophoresis: serum from (C) a patient with $\mathrm{CF}$ with a high antibody titre against $P$ intermedia cell wall proteins (1:4682) and (D) a patient with CF with chronic Pseudomonas aeruginosa infection were subjected to crossed immunoelectrophoresis using the respective $P$ intermedia or $P S$ aeruginosa cell proteins as antigens.
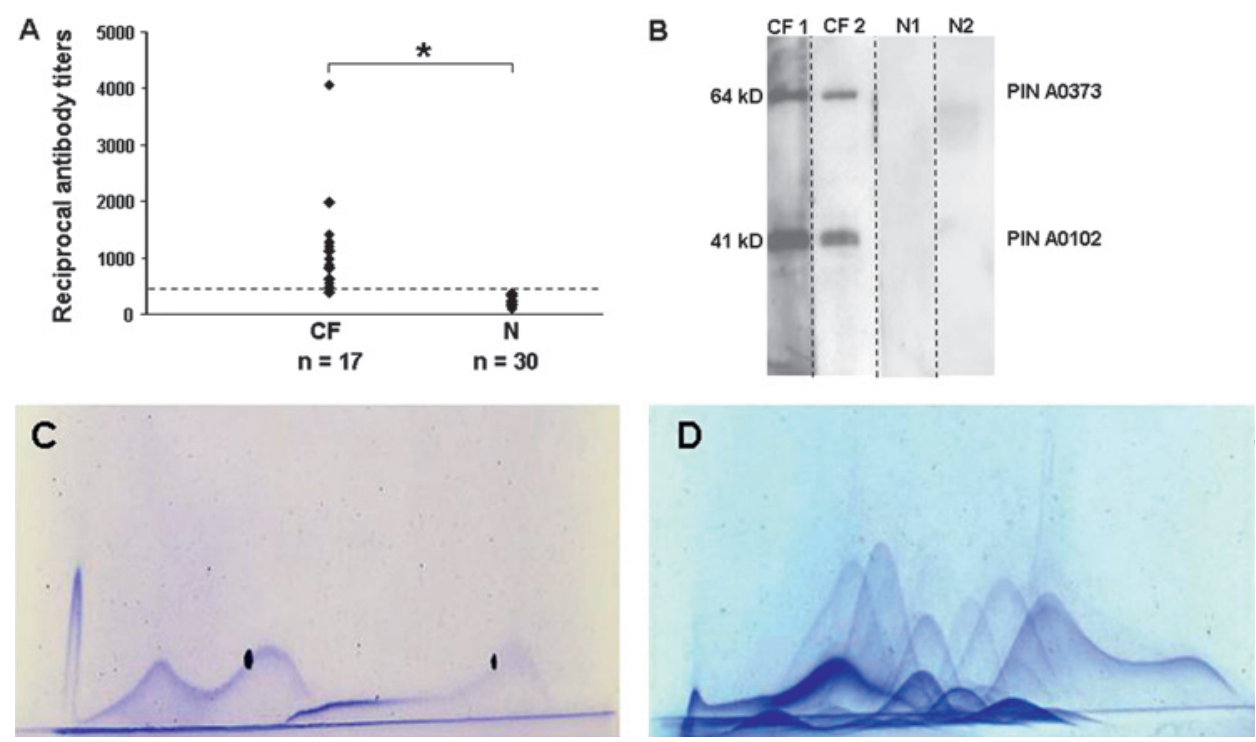
sputum. To exclude the possibility that sputum specimens are contaminated by Prevotella species from oral flora, we quantified these micro-organisms in bronchopulmonary samples collected by fibreoptic bronchoscopy. When bronchopulmonary samples from four patients with CF (median age 11.5 years) were cultured anaerobically on media we found different Prevotella species in numbers of $>10^{5}->10^{7} \mathrm{CFU}$, corroborating the results by Tunney et $a l^{17}$ and suggesting that bacterial growth had occurred within the lower airways rather than contamination of airway samples with the oral anaerobic flora during recovery (table 2).

Culture-independent diagnosis yielded a higher prevalence of Prevotella species in sputum specimens from patients with CF. Not all patients with antibodies against $P$ intermedia had $P$ intermedia recovered from sputum taken at the time the serum was obtained. While this may be due to the persistence of specific antibodies even after eradication of the pathogen, it may also result from a lack of growth of the anaerobes on culture plates (ie, a false negative diagnosis). We therefore tested a culture-independent novel PNA probe for the identification of Prevotella spp. According to the sequence information of the distributor, $P$ intermedia cannot be distinguished from $P$ nigrescens

Table 1 Strains used in the study

\begin{tabular}{|c|c|c|}
\hline Microorganism & Strain & Source \\
\hline \multirow[t]{2}{*}{ Prevotella intermedia } & ATCC 25611 & A \\
\hline & ATCC 20706 & A \\
\hline Prevotella oralis & ATCC 20702 & \\
\hline Prevotella paludivivens & ATCC 17968 & \\
\hline Prevotella nigrescens & ATCC 13386 & \\
\hline Bacteroides fragilis & ATCC 25285 & \\
\hline \multirow[t]{13}{*}{ Prevotella nigrescens } & 138 & B \\
\hline & 582 & B \\
\hline & 621 & B \\
\hline & 634 & B \\
\hline & 655 & B \\
\hline & 658 & B \\
\hline & 672 & B \\
\hline & 1006 & B \\
\hline & 1024 & B \\
\hline & 1032 & B \\
\hline & 1044 & B \\
\hline & 1048 & B \\
\hline & 1057 & B \\
\hline Prevotella oralis & 1243 & B \\
\hline Prevotella oris & 1037 & B \\
\hline \multirow[t]{2}{*}{ Prevotella baroniae } & 651 & B \\
\hline & 1033 & B \\
\hline \multirow[t]{2}{*}{ Prevotella bivia } & 1050 & B \\
\hline & 1071 & B \\
\hline \multirow[t]{2}{*}{ Prevotella buccae } & 578 & B \\
\hline & 674 & B \\
\hline \multirow[t]{2}{*}{ Prevotella denticola } & 583 & B \\
\hline & 608 & B \\
\hline \multirow[t]{2}{*}{ Prevotella disiens } & 1059 & B \\
\hline & 1074 & B \\
\hline \multirow[t]{2}{*}{ Prevotella melaninogenica } & 1029 & B \\
\hline & 1049 & B \\
\hline \multirow[t]{2}{*}{ Prevotella multiformis } & 507 & B \\
\hline & 524 & B \\
\hline Prevotella intermedia & 1045 & B \\
\hline Actinomyces odontolyticus & R2 & B \\
\hline Pseudomonas aeruginosa & PA01 & B \\
\hline Staphylococcus aureus & ATCC 35556 & A \\
\hline
\end{tabular}

A, German Collection of Microorganisms and Cell Cultures (DSZM); B, Institute for Medical Microbiology and Hygiene, Tübingen, Germany; identified by API. by this technique. The specificity of the probe for $P$ intermedia and $P$ nigrescens was confirmed by applying the probe to a large number of other Prevotella strains (table 1). Four other bacterial strains were also used (table 1). Only negative staining results were obtained (data not shown). The probe was applied to 17 sputum samples from patients with CF and identified Prevotella spp. in $47 \%$ of the samples (figure $4 \mathrm{~A}, \mathrm{~B}$ ).

In contrast, growth of Prevotella spp. from sputum specimens on culture plates was only achieved in four cases (23\%). To specify to which species grown colonies belong, we applied MALDI-TOF-MS. As gold standards for detection of Prevotella species by MALDI-TOF-MS, 27 different sequenced Prevotella strains stored in our large strain collection at the Institute of Medical Microbiology and Hygiene were used (table 1). As examples, distinct MALDI-TOF-MS patterns for $P$ intermedia, $P$ nigrescens and $P$ melaninogenica are depicted in figure $4 \mathrm{C}-\mathrm{E}$. Using these references, two of the four Prevotella sputum isolates were identified as $P$. intermedia and the other two as $P$. nigrescens and $P$. melaninogenica, respectively. These results were corroborated by the sequence analysis of the four Prevotella CF isolates (accession numbers: HM998853, HM998854, HM998855, HM998856).

Collectively, these data show that Prevotella species and possibly other obligate anaerobic species present in sputum specimens from patients with CF may be missed by culturedependent methods.

\section{DISCUSSION \\ Contribution of $\boldsymbol{P}$ intermedia to the pathogenicity of lung disease in CF}

In this study we show that $P$ intermedia may contribute to the complex pathophysiology in the chronically infected CF lung. Several lines of evidence support this notion. First, Prevotella species were detected in numbers $>10^{5} \mathrm{CFU}$, showing that bacterial growth had occurred within the lower airways rather than contamination of airway samples with the oral anaerobic flora during recovery (table 2). This result corroborates data from other investigators. ${ }^{17}$

Second, in contrast to healthy individuals, $P$ intermedia antigens are recognised by the adaptive immune system of the vast majority of patients with CF resulting in specific antibody titres, which again argues against contamination of airway samples with the oral anaerobic flora during recovery (figure $3 \mathrm{~A}$ ). The fact that, in some patients, positive serum antibody titres against $P$ intermedia antigens were found in the absence of the microorganism may be explained by either a false negative detection of $P$ intermedia (see below) or the possibility that patients who cleared the microorganism still had circulating antibodies. Similar phenomena occur with $P_{s}$ aeruginosa serum antibody titres and detection of the microorganism in CF airway specimens. ${ }^{30} 31$

Third, extracellular toxins of $P$ intermedia are cytotoxic for human alveolar type II cells and neutrophils (figure 1A, B,C), and they induce the influx of macrophages and neutrophils in the airway lumen thereby increasing lung inflammation (figure 1D, E). Using assays for gelatin, elastin and casein cleavage, we were unable to identify proteases with these activities in culture supernatant fluids of $P$ intermedia strain ATCC 25611 which had pronounced cytotoxicity to A549 cells.

Last, $P$ intermedia possesses a surface protein $^{32}$ which, in analogy to $S$ aureus, ${ }^{33}$ may avoid opsonophagocytosis by binding the Fc portion of human $\operatorname{IgG}$, rendering specific antibodies inefficiently protective (figure $3 \mathrm{~B}$ ).

Importantly, the cytotoxic and inflammatory potential of culture supernatant fluids of $P_{S}$ aeruginosa grown anaerobically 
A

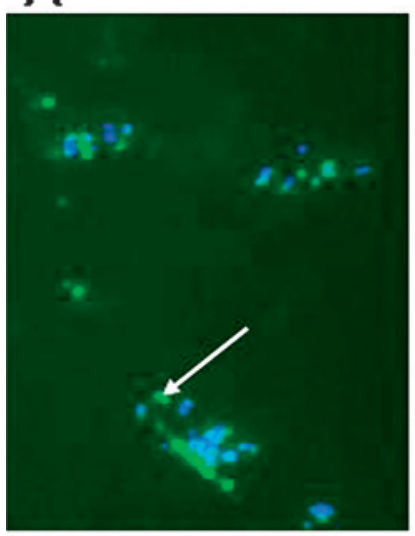

B

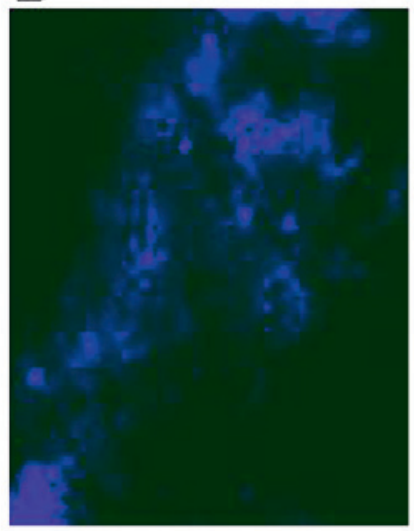

D

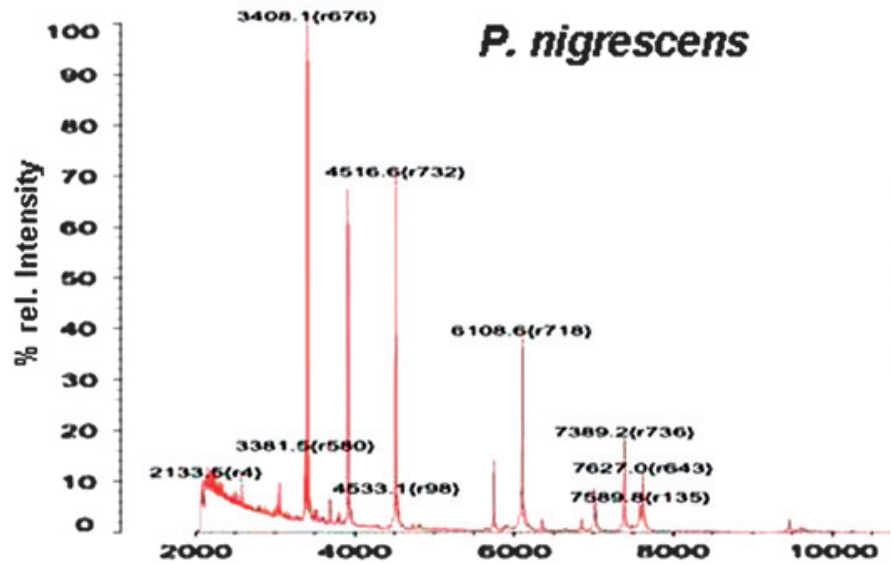

C

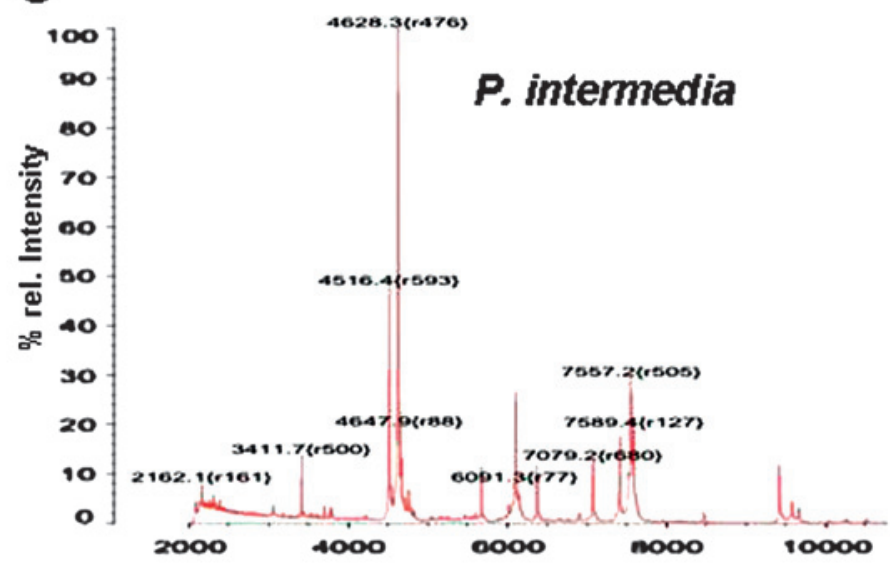

E

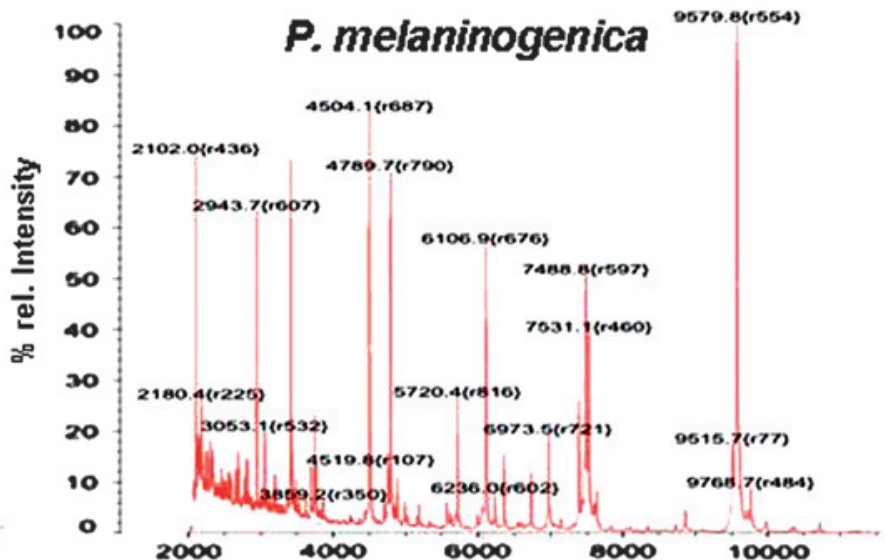

Figure 4 Detection of Prevotella intermedia/Prevotella nigrescens by a novel FITC-labelled PNA probe. Prevotella spp. were stained by the PNA probe in a representative sample of patients with cystic fibrosis. (A,B) DAPI staining. (C-E) MALDI-TOF-MS spectra of (C) $P$ intermedia, (D) $P$ nigrescens and (E) $P$ melaninogenica.

to cell numbers comparable to $P$ intermedia was much lower (figure $1 \mathrm{~A}, \mathrm{D}, \mathrm{E})$. Provided that comparable $P$ intermedia cell numbers are reached in CF airways to those used in our in vitro assays $\left(>10^{8} \mathrm{CFU}\right)$, P intermedia would be more pathogenic than anaerobically grown $P$ s aeruginosa. However, although growth under anaerobic environmental conditions is the dominant life style for Ps aeruginosa in the CF lung $^{34}$ and the its virulence decreases during chronic CF lung infection due to adaptive radiation, ${ }^{35}$ Ps aeruginosa can also multiply in aerobic compartments of the CF lung, in contrast to $P$ intermedia, and contribute to the pathophysiology of CF lung disease. Our animal experiments support this notion.

\section{Detection of Prevotella species in bronchopulmonary samples of patients with CF}

In general, reports on anaerobic bacterial species in CF sputum samples are rare, ${ }^{14-20}$ although growth of obligate anaerobic species in CF sputum specimens may be facilitated by the high viscosity of the sputum ${ }^{2}$ and the presence of facultative anaerobic bacteria which rapidly consume oxygen, thus lowering the oxygen tension to degrees which allow obligate anaerobic bacteria to thrive.

In this study we show that the fluoreszenz in situ hybridisierung (FISH) method for detection of $P$ intermedia and $P$ nigrescens yields better results in this context than the classical API system. By using the novel PNA probe, $47 \%$ of the sputum samples were found positive for $P$ intermedia and/or $P$ nigrescens while only in $23 \%$ of the sputum samples were Prevotella spp. identified by culture and API. One explanation is that we may have missed $P$ intermedia during sputum solubilisation. Our finding may also explain in part why we detected $P$ intermediaspecific serum antibody titres in several $P$ intermedia culturenegative CF patients. Compared with the API system-which is known to have various disadvantages such as interpretation of biochemical reactions, long incubation time periods and cost$\mathrm{s}$ - the novel PNA probe is much more rapid. Furthermore, FISH is more sensitive than API due to different sample processing. While the probe cannot be applied to detect these Prevotella spp. in unprocessed CF sputum, solubilisation with 1,4-Dithiothreitol (DTT) followed by centrifugation and fluorescence microscopy is sufficient to obtain a result within $4 \mathrm{~h}$. The PNA probe may allow rapid screening of $P$ intermedia in the sputum from patients with CF which, in positive cases, may be followed

Table 2 Prevotella species in bronchopulmonary samples of patients with cystic fibrosis

\begin{tabular}{lll}
\hline Patient & Strains & CFU/mI \\
\hline 1 & Prevotella denticola & $>10^{7}$ \\
2 & Prevotella intermedia & $>10^{5}$ \\
3 & Prevotella melaninogenica & $5 \times 10^{6}$ \\
4 & Prevotella melaninogenica & $5 \times 10^{6}$ \\
\hline
\end{tabular}


by a more elaborate and time-consuming quantitative assessment of $P$ intermedia. These data can be used for the control of treatment directed against strict anaerobes.

Taken together, the results of this study suggest that $P$ intermedia plays a critical role in the complex pathophysiology of lung disease in patients with CF when cell numbers of $>10^{9} \mathrm{CFU}$ are reached in the anaerobic sputum plugs.

Acknowledgements The authors thank Stephan Jung, Proteomic Centre, University of Tübingen, Germany for MALDI-TOF analysis of Prevotella intermedia membrane proteins and Mark Fiandaca, AdvanDx, Woburn, Massachusetts, USA for synthesising a Prevotella PNA probe.

Funding Forschungsprogramm der Tübinger Medizinischen Fakultät fortüne-Programm No. 1697-0-0; Universitätsklinikum Tübingen, Germany.

Competing interests None.

Patient consent Obtained

Ethics approval This study was conducted with the approval of the ethic committees of the University of Tübingen, the Hospices Civils de Lyon and the Université Claude Bernard, Lyon, France.

Provenance and peer review Not commissioned; externally peer reviewed.

\section{REFERENCES}

1. Boucher R. New concepts of the pathogenesis of cystic fibrosis lung disease. Eur Respir J 2004:23:146-58.

2. Matsui H, Grubb BR, Tarran R, et al. Evidence for periciliary liquid layer depletion, not abnormal ion composition, in the pathogenesis of cystic fibrosis airways disease. Cell 1998:95:1005-15.

3. Döring G, Ratjen F. Immunology of cystic fibrosis. In: Hodson ME, Geddes D, Bush A, eds. Cystic fibrosis. London: Arnold Hammer, 2007:69-80.

4. Anon. Patient registry annual report. Maryland, USA: Cystic Fibrosis Foundation, 2004.

5. Cramton SE, Ulrich M, Götz F, et al. Anaerobic conditions induce expression of polysaccharide intercellular adhesin in Staphylococcus aureus and Staphylococcus epidermidis. Infect Immun 2001;69:4079-85.

6. Matsui H, Wagner VE, Hill DB, et al. A physical linkage between cystic fibrosis airway surface dehydration and Pseudomonas aeruginosa biofilms. Proc Natl Acad Sci USA 2007;103:18131-6.

7. McKenney D, Pouliot KL, Wang Y, et al. Broadly protective vaccine for Staphylococcus aureus based on an in vivo-expressed antigen. Science 1999:284:1523-7.

8. Worlitzsch D, Tarran R, Ulrich M, et al. Effects of reduced mucus oxygen concentration in airway Pseudomonas infections of cystic fibrosis patients. J Clin Invest 2002;109:317-25.

9. Döring G, Hoiby N; Consensus Study Group. Early intervention and prevention of lung disease in cystic fibrosis: a European consensus. J Cyst Fibros 2004;3:67-91.

10. Hill D, Rose B, Pajkos A et al. Antibiotic susceptibilities of Pseudomonas aeruginosa isolates derived from patients with cystic fibrosis under aerobic, anaerobic, and biofilm conditions. J Clin Microbiol 2005;43:5085-90.

11. Kolpen M, Hansen CR, Bjarnsholt $\mathrm{T}$, et al. The respiratory burst of polymorphonuclear leukocytes accelerates oxygen depletion in sputum from CF patients with chronic Pseudomonas aeruginosa lung infection. Thorax 2010;65:57-62

12. Brook I, Fink R. Transtracheal aspiration in pulmonary infection in children with cystic fibrosis. Eur J Respir Dis 1983:64:51-7.

13. Jewes LA, Spencer RC. The incidence of anaerobes in the sputum of patients with cystic fibrosis. J Med Microbiol 1990;31:271-4.

14. Rogers GB, Hart CA, Mason JR, et al. Bacterial diversity in cases of lung infection in cystic fibrosis patients: $16 \mathrm{~S}$ ribosomal DNA (rDNA) length heterogeneity PCR and
$16 \mathrm{~S}$ rDNA terminal reobligateion fragment length polymorphism profiling. J Clin Microbiol 2003;41:3548-58

15. Rogers GB, Carroll MP, Serisier DJ, et al. Characterization of bacterial community diversity in cystic fibrosis lung infections by use of 16 s ribosomal DNA terminal reobligation fragment length polymorphism profiling. J Clin Microbiol 2004:42:5176-83.

16. Rogers GB, Carroll MP, Serisier DJ, et al. Use of 16S rRNA gene profiling by termina restriction fragment length polymorphism analysis to compare bacterial communities in sputum and mouthwash samples from patients with cystic fibrosis. J Clin Microbiol 2006; 44:2601-4.

17. Tunney MM, Field TR, Moriarty TF, et al. Detection of anaerobic bacteria in high numbers in sputum from patients with cystic fibrosis. Am J Respir Crit Care Med 2008;177:995-1001.

18. Worlitzsch D, Rintelen C, Böhm K, et al. Antibiotic-resistant obligate anaerobes during exacerbations of cystic fibrosis patients. Clin Microbiol Infect 2009;15:454-60

19. Bittar F, Riichet H, Dubus J-C, et al. Molecular detection of multiple emerging pathogens in sputa from cystic fibrosis patients. PloS One 2008;3:e2908.

20. Harris JK, De Groote MA, Sagel SD, et al. Molecular identification of bacteria in bronchoalveolar lavage fluid from children with cystic fibrosis. Proc Natl Acad Sci USA 2007;104:20529-33

21. Gove RI, Wiggins J, Stableforth DE. A study of the use of ultrasonically nebulized lidocaine for local anaesthesia during fiberoptic bronchoscopy. $\mathrm{Br} J$ Dis Chest 1985; 79:49-59.

22. Oliveira K, Procop GW, Wilson D, et al. Rapid identification of Staphylococcus aureus directly from blood cultures by fluorescence in situ hybridization with peptide nucleic acid probes. J Clin Microbiol 2002;40:247-51.

23. Fenselau C, Demirev PA. Characterization of intact microorganisms by MALDI mass spectrometry. Mass Spectrom Rev 2001;20:157-71.

24. Johansen HK, Nørregaard L, Gøtzsche PC, et al. Antibody response to Pseudomonas aeruginosa in cystic fibrosis patients - a marker of therapeutic success? A 30-year cohort study of survival in Danish CF patients after onset of chronic P. aeruginosa lung infection. Pediatr Pulmonol 2004;37:427-32.

25. Ulrich M, Albers C, Moller JG, et al. Moxifloxacin and azithromycin but not amoxicillin protect human respiratory epithelial cells against Streptococcus pneumoniae in vitro when administered up to 6 hours after challenge. Antimicrob Agents Chemother 2005;49:5119-22.

26. Jensen PO, Bjarnsholt T, Phipps R, et al. Rapid necrotic killing of polymorphonuclea leukocytes is caused by quorum-sensing-controlled production of rhamnolipid by Pseudomonas aeruginosa. Microbiology 2007;153:1329-38.

27. Bragonzi A, Worlitzsch D, Timpert P, et al. Non-mucoid Pseudomonas aeruginosa expresses alginate in lungs of patients with cystic fibrosis and in a murine model. $J$ Infect Dis 2005;192:410-19.

28. Brook I. Anaerobic infections in children. Microb Infect 2002;4:1271-80

29. Jansen HJ, Grenier D, van der Hoeven JS. Characterization of immunoglobulin G-degrading proteases of Prevotella intermedia and Prevotella nigrescens. Oral Microbiol Immunol 1995;10:138-45.

30. Döring G, Høiby N. Longitudinal study of immune response to Pseudomonas aeruginosa antigens in cystic fibrosis. Infect Immun 1983;42:197-201.

31. Ratjen F, Walter $\mathrm{H}$, Haug $\mathrm{M}$, et al. Diagnostic value of serum antibodies in early Pseudomonas aeruginosa infection in cystic fibrosis patients. Pediatr Pulmonol 2007:42:249-55.

32. Grenier D, Michaud J. Demonstration of human immunoglobulin G Fc-binding activity in oral bacteria. Clin Diagn Lab Immunol 1994;1:247-9.

33. Graille M, Stura EA, Corper AL, et al. Crystal structure of a Staphylococcus aureus protein $\mathrm{A}$ domain complexed with the Fab fragment of a human IgM antibody: structural basis for recognition of B-cell receptors and superantigen activity. Proc Nat Acad Sci USA 2000;97:5399-404.

34. Döring G, Parameswaran I, Murphy TF. Differential adaptation of microbial pathogens to airways of patients with cystic fibrosis and chronic obstructive pulmonary disease. FEMS Microbiol Rev 2010 May 28. doi:10.1111/j.15746976.2010.00237

35. Smith EE, Buckley DG, Wu Z, et al. Genetic adaptation by Pseudomonas aeruginosa to the airways of cystic fibrosis patients. Proc Natl Acad Sci USA 2006;103:8487-92. 